\title{
Dietary Influence of the Gut Microbiome - Potential Hazards and Benefits
}

\author{
Edward Yang and David A. Johnson*
}

Eastern VA Medical School, Norfolk VA, USA

*Corresponding author: David A. Johnson, Professor of Medicine/Chief GI, Eastern VA Medical School, Gastroenterology, 885 Kempsville Rd Suite 114, Norfolk, VA 23502, United States, Tel: 007576416685; E-mail: dajevms@aol.com

Received date: Nov 12, 2014, Accepted date: Nov 29, 2014, Publication date: Dec 9, 2014

Copyright: (c) 2014 Yang E, et al. This is an open-access article distributed under the terms of the Creative Commons Attribution License, which permits unrestricted use, distribution, and reproduction in any medium, provided the original author and source are credited.

\begin{abstract}
Over the past decade, significant advancements in metagenomics and metabolomics have shed light on the intricate influence of the gut microbiome on the course of systemic diseases. Even more recently, data has emerged on specific bacterial metabolites and antigens interacting with the immune system to modulate these diseases. As diet partly determines the gut flora, we analyzed studies on dietary effects on the gut microbiome and discuss the impact of this interaction on a broad spectrum of systemic diseases. We performed PubMed and Google searches limited to the past 10 years to compile the latest data and trends in dietary influences on the microbiome and systemic diseases. We found that dietary effects on the gut microbiome plays a major role in the course of a variety of systemic diseases including cancer, Inflammatory Bowel Disease (IBD), metabolic syndrome, atherosclerosis, auto-inflammatory disease, and asthma. This is mediated via immunomodulation and bacterial metabolites of dietary sources. Short Chain Fatty Acids (SCFAs) were identified as microbiome metabolites of undigestable polysaccharides affecting broad ranges of diseases using diverse functions in G-protein receptor binding, immunomodulation, hormone regulation, and mucosal protection. Diet and the gut microbiome appear to have interactive roles in influencing a broad range of systemic diseases, but only a finite, very specific number of mechanisms have been identified to date. Data in the near future should focus on expanding our knowledge on bacterial metabolomics as it relates to these systemic diseases.
\end{abstract}

Keywords: Gut microbiome; Microbiota; Metabolomics; Diet; Dietary effects; Inflammatory; Colon; Immune system; Cancer; Atherosclerosis; Asthma; Metabolic syndrome; Nonalcoholic Fatty liver disease; NAFLD; NASH

\section{Introduction}

It is well known that the gut houses the largest immune system in the body [1]. Recently, there has been a focus on the cross-talk between gut immunity and the host microbiome and the subsequent effect of this interaction on a broad range of diseases. More recently, diet has been shown to shape the gut microbiome and alter immune function via the metabolome [1]. This process appears to begin at birth and has a durable effect on the immune profile. Ardeshir et al. demonstrated that breast-fed and bottle-fed infant rhesus macaques developed distinct gut microbiomes by 6 months, with breast-fed macaques having more Prevotella and Ruminococcus and bottle-fed macaques enriched with Clostridium [2]. By 12 months, breast-fed macaques had a 15 -fold higher percentage of Th17 cells within the $\mathrm{CD} 4+\mathrm{T}$ cell population, as well as higher numbers of $\mathrm{CD} 4+$ and CD8+ T cells overall.

Over the past decade, major advancements in metagenomics and metabolomics have allowed us to advance our understanding of the gut microbiome. Recent studies in both mice and humans have helped to characterize the influence of certain genetic, environmental, and dietary factors on specific microbiome profiles. Turnbaugh et al. was amongst the first in 2009 to discover that the germ-free mouse gut could be "humanized" with human gut flora, demonstrating that subsequent switching from a plant-based to "Western" high-fat and sugar diet caused distinct shifts in the gut microbiome within 1 day [3]. This distinct shift in the microbiome profile secondary to a
"Western" diet is also seen in humans and occurs along the same timeline [4]. Additionally, identification of specific bacterial species and how they relate to disease pathology has continued to elucidate the regulatory role the immune system plays in the gut microbiome. Although recognizably understanding the full spectrum of the "gut microbiome's" role is still in a relative infancy, it is clear that our bacterial flora play a much larger role in systemic diseases than previously appreciated. Additionally, there has been recognition that selected dietary intake may favorably influence the gut microbiome by a "prebiotic" effect. Prebiotic is a general term to refer to chemicals that induce the growth and/or activity of commensal microorganisms (e.g., bacteria and fungi) that contribute to the well-being of their host. The most common example is in the gastrointestinal tract, where prebiotics can alter the distribution of organisms in the gut microbiome. A prebiotic is a selectively fermented ingredient that allows specific changes, both in the composition and/or activity in the gastrointestinal microflora that confers benefits upon host well-being and health, but it does not emphasize a specific bacterial group. In this review, we provide an overview of dietary and metabolite influences on a variety of common diseases as they relate to the gut microbiome.

\section{Cancer}

The majority of the diversity in our microbiome is in the colon. There has been recent links between certain pathogenic bacteria and the development of colorectal cancer, and the current thought is that select gut microbiomes can mediate a chronic inflammatory environment which contributes to the progression of colorectal cancer. The lower incidence of colorectal cancer in rural, native Africans compared to African Americans has been attributed to higher amount of undigestible polysaccharides in the diet [5]. Therefore, it is of particular interest to focus on the role of diet and the metabolome 
in the adenoma-carcinoma sequence. Undigestible polysaccharides passing into the gut, namely from dietary fiber, are metabolized by the microbiome into Short Chain Fatty Acids (SCFAs), which are then converted into acetate, propionate, and butyrate [6]. The latter two inhibit intracellular Histone Deacetylases (HDACs), which downregulate pro-inflammatory cytokines IL-6 and IL-12 and induce increased differentiation of CD4+ $\mathrm{T}$ cells into regulatory $\mathrm{T}$ cells (Tregs) [5,7]. The net result is a decrease in the inflammatory mediators in the colon. Extracellular SCFAs have recently been shown to interact with a variety of G-Protein Coupled Receptors (GPRs). The SCFA butyrate signals via GPR109A to increase anti-inflammatory cytokine IL-10, promote Treg differentiation, and inhibit NF-kB [5]. In addition, microbial metabolism of proteins into polyamines and hydrogen sulfide, and fat-induced bile acids into taurine and secondary bile acids, increase the inflammatory factors in the colon. Taken together, an individual's diet and unique microbiome are thought to influence the pro-inflammatory state of the colon via both immunologic and metabolite-mediated mechanisms. This is thought to contribute to progression of the adenoma-carcinoma sequence for colon cancer.

A High-Fat Diet (HFD) has been shown by Schulz, Atay, Heringer et al. to promote small bowel carcinomas through specific microbiome profiles and modulation of the immune system. Mice expressing the oncogene K-ras develop spontaneous serrated hyperplasia in the duodenum and downregulate the Paneth-cell mediated secretion of antimicrobial defensins [8]. The addition of a HFD alters the microbiome in mice and significantly decreased the amount of mucin on the intestinal lining, which serves as a barrier to microbial damage and maintains gut homeostasis [9]. This results in altered immunological and mucinous barriers of the intestinal lumen, which allows increased exposure to potential pathogenic effects of the individual's gut microbiome. When Schulz, Atay, Heringer et al. performed fecal transplants from HFD K-ras mice into healthy K-ras mice, they conferred an elevated risk for small bowel carcinoma, while treatment with antibiotics eliminated HFD-induced carcinomas [8]. Of particular interest, a HFD decreased the amount of SCFAs in the colon, while the administration of butyrate, a SCFA, markedly decreased the incidence of duodenal carcinoma in HFD-mice [8]. There was also a sharp increase in Bifidobacteriaceae and Porphyromonadaceae, decrease in Helicobacteraceae, and partial reversal of the HFD-induced deficits on mucin production and immune function. This suggests a key role of diet in the pathogenesis of intestinal carcinoma via effects on the gut microbiome and immune system.

Outside the gastrointestinal tract, diet and the gut microbiome are postulated to influence the course of human breast cancer through bacterial-mediated metabolism of estrogen and microbiome dependent maturation of CD8+ T cells [10]. Abundant SCFAs in a high-fiber diet increase the number of Firmicutes and Bacteroidetes in the gut microbiome [11]. These microbes metabolize lignans in the diet into potent phytoestrogens that have been shown to inversely affect the risk of breast cancer [10]. In contrast, estrogen is a major hormonal growth promoter of breast cancer that is conjugated in the liver and secreted in the bile for elimination [12]. The microbiome is capable of deconjugating this estrogen, and the increase in estrogen reabsorption can increase the risk of breast cancer [10]. Data suggests that CD8+ T cells are the most potent lymphocytes for killing breast cancer cells, with increased cell numbers in breast tumors correlating to better survival [13]. Data shows that CD8 $+\mathrm{T}$ cell activation into functional effector cells with cytotoxic properties partly depends on contact with Sphingomonas antigens in the small and large intestines [14]. Clearly, more data is needed to further define the gut microbiome's role in the pathogenesis of breast cancer.

\section{Inflammatory Bowel Disease (Ibd)}

As data on the gut microbiome, metabolomics, and dietary influences on IBD continues to grow [15], the causal relationship between the dietary effects of the microbiome on IBD still remains unclear. Much of what we know thus far comes from studies in murine models of colitis as it relates to dysfunction of the immune system. Mice deficient in IL-10, an anti-inflammatory cytokine, develop spontaneous colitis secondary to massive T-Helper 1 (Th1) cell activation, but this colitis is prevented when mice are reared in germfree environments, which eliminates the role of the gut microbiome [1]. When germ-free IL-10 deficient mice are exposed to specific gut flora, different severities of colitis can be induced [1]. These studies suggest an important role for the gut microbiome in IBD.

Devkota et al. demonstrated that a high milk-fat diet elicited a blooming population of Bilophilia wadsworthia in IL-10 deficient mice and promoted the development of Th1-mediated colitis. Milk fats increase hepatic conjugation of taurine to bile acids, which provides B. wadsworthia with a sulfur source for growth [16]. Supplementation of taurine-conjugated bile acids to the diet alone was sufficient to increase the population of B. wadsworthia in the microbiome and incidence of colitis in IL-10 deficient mice. Though the precise antigen triggering colitis was never isolated, the presence of B. wadsworthia induced a cytokine profile consistent with a Th1 response. Humans with Ulcerative Colitis (UC) demonstrate increased gut sulfurreducing bacteria as well as production of hydrogen sulfide, which correlates with the increased B. wadsworthia in the work of Devkota et al. It has also been suggested that hydrogen sulfide acts as an oxidizing agent that further promotes the pro-inflammatory environment typical in IBD [17]. Of particular interest, David et al. found that a high fat diet rapidly shifted the gut microbiome in healthy humans to an abundant increase in B. wadsworthia, again consistent with the findings of Devkota et al. [4]. In addition, they also found that the high animal fat diet significantly increased bile acid production and induced microbial DNA and RNA encoding sulphite reductases. Though they did not look for IBD related symptoms, this data provides a solid groundwork for future human studies on the role of the diet and microbiome on IBD.

Another metabolomic change found by David et al. was that a high fat diet also decreased the amount of SCFAs produced from undigestible polysaccharides by the gut microbiome, in particular butyrate [4]. As mentioned with cancer, SCFAs have an immunomodulatory effect that decreases the amount of NF-kB and inflammatory cytokines, which is thought to also contribute to the inflammatory environment surrounding IBD. Indeed it has been shown in mice that SCFAs expand the colonic population of Treg cells, which exert anti-inflammatory activity via IL-10 [18]. Using a murine model for colitis, dietary supplementation with SCFAs decreased the severity of colitis. Finally, more recent data has emerged on dietary effects on the Aryl Hydrocarbon receptor (AhR), which functions as a detoxification pathway in the gut [19]. 1,4-Dihydroxy-2-Naphthoic Acid (DHNA), a metabolite of Propionibacterium freudenreichii ET-3 found in cheese, activates AhR and induces an immunomodulary effect by activating IELs and innate lymphoid cells to produce antimicrobial C-type lectin RegIII [19]. In another mouse model for chemically induced colitis, supplementation with DHNA reduced 
colitis severity by modulating the gut microbiome. While the field of dietary and microbiome influences on IBD is still young, there appears to be compelling data correlating with human findings to justify pursuit for further therapeutic interventions that target this axis.

\section{Metabolic Syndrome and Insulin Resistance}

By recent estimates, the obesity epidemic in the United States has an annualized cost of $\$ 147$ billion [20]. As of 2012, obesity in the United States plagued $34.9 \%$ of adults 20 years or older, as well as $8.1 \%$ of infants and toddlers also fell into this category [21]. Obesity-related increased incidences of atherosclerotic vascular disease, diabetes mellitus, and non-alcoholic fatty liver disease are some of the most common preventable causes of death in the United States. With the close relationship between obesity and diet, it is important to investigate the role of the gut microbiome in the propagation of this epidemic. In mice, transfer of the gut microbiome in genetically obese mice into germ-free mice resulted in transmissible increase in body fat and insulin resistance within 2 weeks [22]. This suggested that the gut flora could, in part, dictate the phenotype of obesity in a transmissible fashion. As discussed earlier, bacterial degradation of undigestible polysaccharides is crucial in the generation of SCFAs and its metabolites. Lin et al. demonstrated that SCFAs regulate gut hormones that control satiety by binding to Free Fatty Acid Receptors 2 (FFAR2) and 3 (FFAR3), which are also GPRs [23]. They found that the SCFAs butyrate and propionate reduced overall food intake via FFAR3. It has previously been shown that SCFAs induce the gut hormones Glucagon-Like Peptide (GLP) and Peptide YY (PYY), which mediate satiety by modulating the release of digestive enzymes [24]. FFAR2 stimulates fat deposition and works with FFAR3 to regulate intestinal motility and satiety. Murine deficiencies in either FFAR-2 or 3 resulted in significantly less body fat mass, increased lean body mass, and lower triglyceride and cholesterol levels [25]. Therefore, the gut microbiome may affect FFARs via SCFA production, modulating the host's energy homeostasis and state of obesity.

A recent study by Suez et al. demonstrated that the same artificial sweeteners that have become a weight-loss staple in today's Western diet can induce glucose intolerance via alterations of the gut microbiome, in both mice and humans [26]. In non-genetically altered mice, both lean and HFD-fed groups developed marked glucose intolerance compared to controls 11 weeks after consuming any of the three Non-Caloric Artificial Sweeteners (NAS) tested [26]. Treatment with antibiotics eliminated this difference, and fecal transplant of flora from NAS-fed mice into germ-free mice elicited glucose intolerance within just 6 days. Metagenomic mapping to a gut microbial gene catalog revealed marked overexpression of glycan degradation pathways for SCFA production, which have previously been linked to increased energy harvest and obesity in both mice and humans [27]. In NAS-fed mice, increases in stool SCFAs propionate and butyrate were found, and this was attributed to upregulation of pathways in five Bacteriodes species [26]. Glucose intolerance was a direct result of NAS-mediated alterations in the microbiome. Feces from non-NAS exposed mice were cultured in NAS-rich conditions and transferred into germ-free mice. This resulted in significant glucose intolerance and an overrepresentation of Bacteriodes species, consistent with metagenomic analysis [26]. In seven healthy human volunteers without NAS consumption, NAS exposure for a short 7-day course developed marked glucose intolerance in four of the seven individuals, with average blood glucose exceeding $150 \mathrm{mg} / \mathrm{dl}$ after a glucose tolerance test [26]. Interestingly, the time course is consistent with that observed recently by David et al. where diet altered the human microbiome within just one day [4]. Fecal transfer of those four responders into germ-free mice also induced significant glucose intolerance. Elegantly that while NAS are touted as a safe method for reducing caloric intake and glucose intolerance, the more global effects of usage warrants reevaluation for safety. The detrimental impact of NAS on the gut microbiome can alter glucose metabolism in as little as a week [26].

\section{Nonalcoholic fatty liver disease}

Parallel to the obesity and metabolic syndrome epidemic in the United States is the increasing incidence of Nonalcoholic Fatty Liver Disease (NAFLD), which comprises of a spectrum of diseases including Nonalcoholic Hepatic Steatosis (NASH). As detrimental sequelae include portal hypertension, hepatocellular carcinoma, and cirrhosis, NAFLD is currently the third leading cause for liver transplantation in the United States [28]. Obesity, insulin resistance, and inflammatory immune processes appear to be the primary risk factors in NAFLD [28]. The excess of free fatty acids (FFAs) in obesity contribute to hepatic steatosis and insulin resistance in type 2 diabetes adds an additional 5-9 fold increase in risk for NAFLD [29-31]. While the gut microbiome's influence on obesity and insulin resistance was discussed earlier, recent data has focused on a more direct role of the interaction between the immune response and the microbiome on the progression of NAFLD.

The innate immune system is reliant on an intrinsic capacity to rapidly detect and eliminate invading foreign pathogenic microbes. The discovery of Toll-like receptors (TLRs) provided a class of membrane receptors which sense extracellular microbes and trigger anti-pathogen signaling cascades [32]. Multiple intracellular microbial sensors have been identified, including NOD-Like Receptors (NLRs). Some of the NLRs also sense non-microbial danger signals and form large cytoplasmic complexes called inflammasomes, which link the sensing of microbial products and metabolic stress to the proteolytic activation of the pro-inflammatory cytokines IL-1beta and IL-18 [32]. The NALP3 inflammasome has been associated with several autoinflammatory conditions including gout [33]. Four TLRs have been identified in the progression of NAFLD: TLR2 recognizing peptidoglycan and lipoteichoic acid on cell walls, TLR4 for lipopolysaccharide, TLR5 for bacterial flagellin, and TLR9 for bacterial DNA [34].

With the microbiota known for playing a role in the development of metabolic syndrome, Mejia et al. fed mice a Methacholine-CholineDeficient Diet (MCDD) to induce NAFLD and found that the inflammasomes NLRP3 and 6 modulate the gut microbiome through IL-18 to decrease disease by reducing portal circulation levels of bacterial TLR4 and 9 agonists [35]. The inflammasome complex is triggered by bacterial antigens and serves to upregulate inflammatory cytokines IL-1 and IL-18 to combat invading microorganisms. When fed a MCDD, mice developed a dramatic increase in Porphyromonadaceae, but mice genetically deficient in the inflammasome pathway developed NAFLD that regressed with antibiotic treatment [35]. They argue that microbiome antigens are first transferred to the liver via the portal-systemic shunt, where they can mediate the effects of NAFLD.

With the involvement of TLRs in NAFLD, it has also been shown that a HFD in mice creates a pro-inflammatory environment that promotes obesity via TLR4 inducing increased intestinal permeability 
and blood endotoxin levels [36]. Interestingly, HFD-fed TLR4deficient mice appear to be protected from NAFLD [37-39]. Several other murine studies have shown that injection of LPS into NAFLD afflicted mice further promotes hepatic damage via elevated proinflammatory cytokines [40,41]. In addition, elevated LPS levels have been found in humans with metabolic syndrome and NAFLD [34]. Overall, it appears that the profile of antigens delivered to the liver may be regulated by both the immune system and one's dietary intake, which suggests a role for the microbiome in NAFLD. This is of particular importance and relevance given that metabolic syndrome has become a rampant condition in Western society, and NAFLD is a common sequelae, as up to $20 \%$ of these patients progress to cirrhosis, portal hypertension, and hepatocellular carcinoma [42].

\section{Atherosclerosis}

The high-fat, low-fiber "Western" diet has also been linked to multiple inflammatory diseases outside of the gastrointestinal tract. It is well known that a high-fat diet is a significant risk factor for atherosclerosis, but one can postulate that there may be a role for the gut microbiome in processing this diet and promoting atherosclerosis. Wang et al. found that Phosphatidylcholine (PC) metabolites produced by the gut microbiome promoted Cardiovascular Disease (CVD) [43]. Foods rich in PC include eggs, milk, liver, red meat, poultry, shell fish, and fish. Mass spectrometry of plasma samples from 75 CVD patients who had subsequently experienced myocardial infarction, stroke, or death identified the presence of Trimethylamine N-Oxide (TMAO) as a significant risk factor [43]. The authors demonstrated via mass spectrometry that PC is converted into Trimethyl Amine (TMA) by the gut flora, and TMA is metabolized into TMAO by hepatic Flavin-Containing Monooxygenases (FMO). In mice susceptible to atherosclerosis, TMAO supplementation upregulated macrophage scavenger receptors linked to atherosclerosis and promoted atherosclerosis [43]. While these studies were performed in mice, Wang et al. interestingly noted that TMAO and TMA levels were elevated in patients on consistent hemodialysis, which correlates with the increased risk for CVD in end-stage renal disease.

In a follow-up study, Two prospective clinical studies that confirmed the gut microbiome dependent production of TMAO in humans and identified TMAO as a risk factor for a major adverse cardiovascular event [44]. In healthy subjects, a TMAO "challenge" consisting of ingestion of two hardboiled eggs and deuterium [d9]labeled PC resulted in increased plasma levels of TMAO. Treatment with broad-spectrum antibiotics eliminated this finding, while subsequent trials post-antibiotics resulted in a variable recovery of microbiome-dependent production of TMAO. In 4,007 patients undergoing cardiac catheterization but without evidence of acute coronary syndrome, baseline TMAO levels were a major predictor of significant adverse cardiovascular events after a 3-year follow-up, even after adjusting for traditional CVD risk factors [44].

Compared to a Western diet, the Mediterranean diet, particularly low in red-meat consumption, is known to decrease the risk for CVD. Along the same TMAO pathway, Koeth et al. showed that gut floral metabolism of L-carnitine, an abundant nutrient in red meat, produced TMAO that accelerated atherosclerosis in both mice and humans with CVD [45]. L-carnitine is a type of TMA that is converted into TMAO by the gut microbiome, and dietary habits are capable of enhancing this process via increased numbers of Prevotella in the gut. In a cohort of 2,595 patients undergoing elective cardiac evaluation, L- carnitine was a risk factor for cardiovascular events by 3 -years in those that had a concurrent elevation of TMAO. Taken a step further, the TMAO-mediated decrease in reverse cholesterol transport and altered cholesterol elimination via bile acid synthesis as mechanisms for increased atherosclerosis [45]. These series of studies provides evidence for a distinct role of the gut microbiome and diet in altering the course of atherosclerosis. While further studies in humans are necessary, there appears to be an encouraging future to further unveiling the influence of the gut microbiome outside of the gastrointestinal tract.

\section{Auto-inflammatory diseases/Asthma}

In the past few decades, there has been a dramatic increase in chronic inflammatory diseases such as asthma, allergies, atherosclerosis, and autoimmune disorders. While theories such as the hygiene hypothesis have prevailed, recent breakthroughs in metagenomics have allowed us to shed light on the gut microbiome's intricate role in modulating these diseases [46]. A protective role for a High Fat Diet (HFD) using a genetic murine model of chronic osteomyelitis (Pstpip2cmo) mediated by IL-1B that closely resembles the disease in humans. Pstpip2cmo mice fed a Low Fat Diet (LFD) developed spontaneous osteomyelitis, measured by hind paw inflammation and bone erosion [47]. The gut microbiome of LFD mice showed an outgrowth of Prevotella and a marked reduction in Lactobacillus, and fecal transplantation of this microbiome into young Pstpip2cmo mice resulted in a significantly accelerated disease course. In contrast, fecal transplant from HFD mice into LFD mice or use of broad-spectrum antibiotics resulted in significant protection from disease [47]. Though a specific metabolite was not identified, Lukens et al. provides reason to suspect a prominent role for the diet and gut microbiome in the course of inflammatory diseases.

Along with autoinflammatory conditions, asthma is another disease that continues to have a rising incidence. While asthma is affected by both polygenic and environmental factors, recent human asthma studies have identified dysbiosis in both the gut and pulmonary flora, showing shifts in the Firmicutes: Bacteroides ratios that suggest a role for the gut microbiome in the pathogenesis of asthma [48]. Asthma is exacerbated in germ-free murine models for asthma, while subsequent bacterial introduction palliates symptoms [49]. Treatment of neonatal asthmatic mice with vancomycin also resulted in increased symptoms [48]. In germ-free asthmatic mice that invariant natural killer T cells (iNKT cells) accumulated in the mucosa of both colon and lung in affected mice [50]. When conventionalized, these mice downregulated CXCL16, a chemokine for iNKT recruitment, in both colon and lung and had decreased iNKT cell infiltration and tissue inflammation. Altogether, these studies suggest that the microbiome can influence the pathogenesis of asthma.

Dietary fiber's effect on the gut microbiome as a factor in allergic airway disease [51]. In particular, their model utilized wild-type mice consuming either low $(<0.3 \%)$, standard $(4 \%)$, or high fiber diets and induced Allergic Airway Inflammation (AAI) via house dust mite (HDM) extract exposure. Of note, their results may approximate more physiological conditions by using a standardized fiber diet and avoiding genetic manipulation. Increased AAI on low fiber diets was measured by increases in inflammatory cytokines IL-4, IL-5, IL-13, and IL-17A, total IgE and HDM-specific IgG1, histological analysis showing increased inflammatory cells, goblet cell hyperplasia, mucus production, and airway hyper-reactivity via methacholine challenge [51]. Dietary fiber increased SCFA production via Bacteroidetes, 
consistent with data discussed earlier. They concluded that SCFA propionate activated Dendritic Cell (DC) specific GPR41 (also known as FFAR3 as discussed in obesity) and impaired activation [51]. The downstream effect is an impaired Th2 response, which translated to decreased AAI symptoms. Substitution of dietary fiber with propionate in HDM exposed mice curbed airway inflammation, suggesting the gut microbiome also plays a unique role in asthmatic diseases that can be influenced by one's diet.

\section{Discussion}

Over the past decade, we have made strides in metagenomics and metabolomics that have allowed us to begin uncovering the vast roles the gut microbiome plays in a broad range of systemic disease. It is clear that diet distinctly and quickly alters the gut microbiome within one day, which leads us to question the extent of how harmful a Western diet is versus the health benefits of a high fiber diet $[3,4]$. While clearly many more studies need to be performed, we have provided evidence in this review that diet and microbiome may be significant potential therapeutic targets in a broad spectrum of diseases ranging from cancer to IBD, metabolic syndrome, asthma, and even atherosclerosis. For example, the recent use of fecal transplantation in the treatment of refractory human Clostridium difficile infection has proven to be effective, durable, and tremendously cost efficient over the current standards in care, oral vancomycin and fidaxomicin [52].

In cancer, particularly of the gastrointestinal tract, a proinflammatory environment mediated contributes to progression of the carcinoma sequence. HFD has been shown to influence the inflammatory environment through a balanced effect on the gut microbiome, which has also been shown to affect the immune system [8]. In addition, specific bacterial species promoted by a high fiber diet produce SCFAs that have demonstrated a protective effect against cancer progression through anti-inflammatory as well as mucinous barrier functions [9]. In mouse models of colitis, diet has been shown to select for specific gut flora that elicit a massive Th1 immune response in IL-10 deficient mice to induce colitis [16]. Interestingly, the same bacteria have been found in patients with ulcerative colitis. In addition, the bacterial metabolite DHNA binding AhR and undigestable polysaccharides that are converted to SCFAs by the gut microbiome promote immunomodulatory effects that decrease inflammation and symptoms of colitis [1].

Obesity and the metabolic syndrome are clearly devastating systemic diseases and a heavy drain on American medical and monetary resources. There appears to be a prominent role of the gut microbiome, as transfer of gut flora from genetically obese mice into non-obese germ free mice is able to confer obesity [22]. Bacterial metabolite SCFAs have been shown in mice to influence gut hormones GLP and PYY to affect satiety and energy metabolism via FFARs [23-25]. In addition, diet also selects for gut microbiomes that can mediate NAFLD/NASH via antigen exposure to the immune system [35]. Lastly, a recent study demonstrated that consumption of popular NAS induces glucose intolerance in both humans and mice within just one week via alterations in the gut microbiome [26]. With the widespread epidemic of metabolic syndrome, further work in dietary effects on the microbiome will be a promising route for future therapies.

Diet and the gut microbiome also impact systemic inflammatory diseases outside of the gastrointestinal system. In cardiovascular disease, a series of studies demonstrated that Phosphatidylcholine
(PC), which is rich in the "Western" diet, increased atherosclerosis by elevating TMAO levels via gut microbiome dependent mechanisms [43]. L-carnitine, rich in red meats, is converted into TMAO by specific gut flora, which was shown to be an independent risk factor for cardiovascular events in patients with elevated TMAO [44,45]. As an interesting contrast in another inflammatory disease, a HFD in mice has been shown to be protective against a genetic model for chronic osteomyelitis by gut microbiome dependent mechanisms [47]. Moving to asthma, germ-free and vancomycin treated mice have exacerbated symptoms while re-introduction of gut flora mitigates symptoms partly by decreasing iNKT cell infiltration [48-50]. Dietary fiber intake increased bacterially converted SCFA propionate that downregulates inflammatory mediators through activation of GPRs on dendritic cells [51]. The end result was decreased airway inflammation. Together, there appears to be evidence for dietary modulation of the gut microbiome being capable of affecting the course of inflammatory diseases.

While the field of the gut microbiome and dietary metabolomics is still young, we have provided specific evidence and series of studies that demonstrated a role for bacterial metabolites in governing disease. Specifically, the effects of bacterial metabolite SCFAs vary broadly from mucosal protection to immunomodulatory to hormone regulation while molecules producing TMAO impact cardiovascular events (Figure 1). However, the data shown here with two metabolites affecting systemic diseases is theoretically just a microcosm of the vast therapeutic potential that lies in the balance between diet and the gut microbiome. There is a clear role of the gut microbiome in the pathogenesis of systemic diseases, but future studies should begin shifting focus towards discovery of bacterial regulated dietary metabolites. Further research is clearly needed to identify how therapeutic manipulations via prebiotic effects can optimize an everemerging number of diseases that have a pathogenic effect via the gut microbiome.

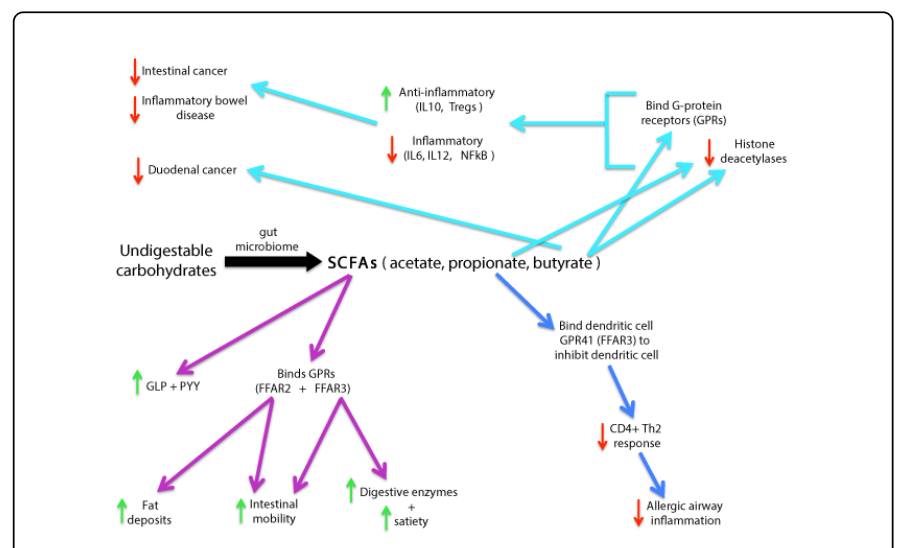

Figure 1: Effects of Bacterial Metabolite SCFAs

\section{References}

1. Leone VA, Cham CM, Chang EB (2014) Diet, gut microbes, and genetics in immune function: can we leverage our current knowledge to achieve better outcomes in inflammatory bowel diseases? Curr Opin Immunol 31C: $16-23$.

2. Ardeshir A, Narayan NR, Mendez-Lagares G, Lu D, Rauch M, et al. (2014) Breast-fed and bottle-fed infant rhesus macaques develop distinct gut microbiotas and immune systems. Sci Transl Med 6: 252ra120. 
3. Turnbaugh PJ, Ridaura VK, Faith JJ, Rey FE, Knight R, et al. (2009) The effect of diet on the human gut microbiome: a metagenomic analysis in humanized gnotobiotic mice. Sci Transl Med 1: 6ra14.

4. David LA, Maurice CF, Carmody RN, Gootenberg DB, Button JE, et al. (2014) Diet rapidly and reproducibly alters the human gut microbiome. Nature 505: 559-563.

5. Louis P, Hold GL, Flint HJ (2014) The gut microbiota, bacterial metabolites and colorectal cancer. Nat Rev Microbiol 12: 661-672.

6. Flint HJ, Scott KP, Duncan SH, Louis P, Forano E (2012) Microbial degradation of complex carbohydrates in the gut. Gut Microbes 3: 289-306.

7. Chang PV, Hao L, Offermanns S, Medzhitov R (2014) The microbial metabolite butyrate regulates intestinal macrophage function via histone deacetylase inhibition. Proc Natl Acad Sci U S A 111: 2247-2252.

8. Schulz MD, Atay C, Heringer J, Romrig FK, Schwitalla S, et al. (2014) High-fat-diet-mediated dysbiosis promotes intestinal carcinogenesis independently of obesity. Nature 514: 508-512.

9. Shan M, Gentile M, Yeiser JR, Walland AC, Bornstein VU, et al. (2013) Mucus enhances gut homeostasis and oral tolerance by delivering immunoregulatory signals. Science 342: 447-453.

10. Shapira I, Sultan K, Lee A, Taioli E (2013) Evolving concepts: how diet and the intestinal microbiome act as modulators of breast malignancy. ISRN Oncol 2013: 693920.

11. Benus RF, van der Werf TS, Welling GW, Judd PA, Taylor MA, et al. (2010) Association between Faecalibacterium prausnitzii and dietary fibre in colonic fermentation in healthy human subjects. Br J Nutr 104: 693-700.

12. Plottel CS, Blaser MJ (2011) Microbiome and malignancy. Cell Host Microbe 10: 324-335.

13. Gritzapis AD, Voutsas IF, Lekka E, Tsavaris N, Missitzis I, et al. (2008) Identification of a novel immunogenic HLA-A*0201-binding epitope of HER-2/neu with potent antitumor properties. J Immunol 181: 146-154.

14. Mercier BC, Ventre E, Fogeron ML, Debaud AL, Tomkowiak M, et al (2012) NOD1 cooperates with TLR2 to enhance T cell receptor-mediated activation in CD8 T cells. PLoS One 7: e42170.

15. Jostins L, Ripke S, Weersma RK, Duerr RH, McGovern DP, et al. (2012) Host-microbe interactions have shaped the genetic architecture of inflammatory bowel disease. Nature 491: 119-124.

16. Devkota S, Wang Y, Musch MW, Leone V, Fehlner-Peach H, et al. (2012) Dietary-fat-induced taurocholic acid promotes pathobiont expansion and colitis in Il10-/- mice. Nature 487: 104-108.

17. Attene-Ramos MS, Nava GM, Muellner MG, Wagner ED, Plewa MJ, et al. (2010) DNA damage and toxicogenomic analyses of hydrogen sulfide in human intestinal epithelial FHs 74 Int cells. Environ Mol Mutagen 51: 304-314.

18. Smith PM, Howitt MR, Panikov N, Michaud M, Gallini CA, et al. (2013) The microbial metabolites, short-chain fatty acids, regulate colonic Treg cell homeostasis. Science 341: 569-573.

19. Fukumoto S, Toshimitsu T, Matsuoka S, Maruyama A, Oh-Oka K, et al. (2014) Identification of a probiotic bacteria-derived activator of the aryl hydrocarbon receptor that inhibits colitis. Immunol Cell Biol 92: 460-465.

20. Finkelstein EA, Trogdon JG, Cohen JW, Dietz W (2009) Annual medical spending attributable to obesity: payer-and service-specific estimates. Health Aff (Millwood) 28: w822-831.

21. Ogden CL, Carroll MD, Kit BK, Flegal KM (2014) Prevalence of childhood and adult obesity in the United States, 2011-2012. JAMA 311: 806-814.

22. Bäckhed F, Ding H, Wang T, Hooper LV, Koh GY, et al. (2004) The gut microbiota as an environmental factor that regulates fat storage. Proc Natl Acad Sci U S A 101: 15718-15723.

23. Lin HV, Frassetto A, Kowalik EJ Jr, Nawrocki AR, Lu MM, et al. (2012) Butyrate and propionate protect against diet-induced obesity and regulate gut hormones via free fatty acid receptor 3-independent mechanisms. PLoS One 7: e35240.
24. Conterno L, Fava F, Viola R, Tuohy KM (2011) Obesity and the gut microbiota: does up-regulating colonic fermentation protect against obesity and metabolic disease? Genes Nutr 6: 241-260.

25. Parekh PJ, Arusi E, Vinik AI, Johnson DA (2014) The role and influence of gut microbiota in pathogenesis and management of obesity and metabolic syndrome. Front Endocrinol (Lausanne) 5: 47.

26. Suez J, Korem T, Zeevi D, Zilberman-Schapira G, Thaiss CA, et al. (2014) Artificial sweeteners induce glucose intolerance by altering the gut microbiota. Nature 514: 181-186.

27. Schwiertz A, Taras D, Schafer K, Beijer S, Bos NA, et al. (2010) Microbiota and SCFA in lean and overweight healthy subjects. Obesity (Silver Spring) 18: 190-195.

28. Machado MV, Cortez-Pinto H (2014) Non-alcoholic fatty liver disease: what the clinician needs to know. World J Gastroenterol 20: 12956-12980.

29. Machado M, Marques-Vidal P, Cortez-Pinto H (2006) Hepatic histology in obese patients undergoing bariatric surgery. J Hepatol 45: 600-606.

30. Anstee QM, Targher G, Day CP (2013) Progression of NAFLD to diabetes mellitus, cardiovascular disease or cirrhosis. Nat Rev Gastroenterol Hepatol 10: 330-344.

31. Targher G, Bertolini L, Padovani R, Rodella S, Tessari R, et al. (2007) Prevalence of nonalcoholic fatty liver disease and its association with cardiovascular disease among type 2 diabetic patients. Diabetes Care 30: 1212-1218.

32. Martinon F, Mayor A, Tschopp J (2009) The inflammasomes: guardians of the body. Annu Rev Immunol 27: 229-265.

33. Martinon F, Petrilli V, Mayor A, Tardivel A, Tschopp J (2006) Goutassociated uric acid crystals activate the NALP3 inflammasome. Nature 440: 237-241.

34. Miura K, Ohnishi H (2014) Role of gut microbiota and Toll-like receptors in nonalcoholic fatty liver disease. World J Gastroenterol 20: 7381-7391.

35. Henao-Mejia J, Elinav E, Jin C, Hao L, Mehal WZ, et al. (2012) Inflammasome-mediated dysbiosis regulates progression of NAFLD and obesity. Nature 482: 179-185.

36. Kim KA, Gu W, Lee IA, Joh EH, Kim DH (2012) High fat diet-induced gut microbiota exacerbates inflammation and obesity in mice via the TLR4 signaling pathway. PLoS One 7: e47713.

37. Rivera CA, Adegboyega P, van Rooijen N, Tagalicud A, Allman M, et al. (2007) Toll-like receptor-4 signaling and Kupffer cells play pivotal roles in the pathogenesis of non-alcoholic steatohepatitis. J Hepatol 47: 571-579.

38. Csak T, Velayudham A, Hritz I, Petrasek J, Levin I, et al. (2011) Deficiency in myeloid differentiation factor-2 and toll-like receptor 4 expression attenuates nonalcoholic steatohepatitis and fibrosis in mice. Am J Physiol Gastrointest Liver Physiol 300: G433-441.

39. Poggi M, Bastelica D, Gual P, Iglesias MA, Gremeaux T, et al. (2007) $\mathrm{C} 3 \mathrm{H} / \mathrm{HeJ}$ mice carrying a toll-like receptor 4 mutation are protected against the development of insulin resistance in white adipose tissue in response to a high-fat diet. Diabetologia 50:1267-1276.

40. Imajo K, Fujita K, Yoneda M, Nozaki Y, Ogawa Y, et al. (2012) Hyperresponsivity to low-dose endotoxin during progression to nonalcoholic steatohepatitis is regulated by leptin-mediated signaling. Cell Metab 16: 44-54.

41. Kudo H, Takahara T, Yata Y, Kawai K, Zhang W, et al. (2009) Lipopolysaccharide triggered TNF-alpha-induced hepatocyte apoptosis in a murine non-alcoholic steatohepatitis model. J Hepatol 51: 168-175.

42. Sheth SG, Gordon FD, Chopra S (1997) Nonalcoholic steatohepatitis. Ann Intern Med 126: 137-145.

43. Wang Z, Klipfell E, Bennett BJ, Koeth R, Levison BS, et al. (2011) Gut flora metabolism of phosphatidylcholine promotes cardiovascular disease. Nature 472: 57-63.

44. Tang WH, Wang Z, Levison BS, Koeth RA, Britt EB, et al. (2013) Intestinal microbial metabolism of phosphatidylcholine and cardiovascular risk. N Engl J Med 368: 1575-1584. 
Citation: Yang E, Johnson DA (2014) Dietary Influence of the Gut Microbiome - Potential Hazards and Benefits. J Nutr Disord Ther 5: 152. doi: 10.4172/2161-0509-1000152

Page 7 of 7

45. Koeth RA, Wang Z, Levison BS, Buffa JA, Org E, et al. (2013) Intestinal microbiota metabolism of L-carnitine, a nutrient in red meat, promotes atherosclerosis. Nat Med 19: 576-585.

46. Thorburn AN, Macia L, Mackay CR (2014) Diet, metabolites, and "western-lifestyle" inflammatory diseases. Immunity 40: 833-842.

47. Lukens JR, Gurung P, Vogel P, Johnson GR, Carter RA, et al. (2014) Dietary modulation of the microbiome affects autoinflammatory disease. Nature .

48. Arrieta MC, Finlay B (2014) The intestinal microbiota and allergic asthma. J Infect 69 Suppl 1: S53-55.

49. Herbst T, Sichelstiel A, Schar C, Yadava K, Bürki K, et al. (2011) Dysregulation of allergic airway inflammation in the absence of microbial colonization. Am J Respir Crit Care Med 184: 198-205.
50. Olszak T, An D, Zeissig S, Vera MP, Richter J, et al. (2012) Microbial exposure during early life has persistent effects on natural killer $\mathrm{T}$ cell function. Science 336: 489-493.

51. Trompette A, Gollwitzer ES, Yadava K, Sichelstiel AK, Sprenger N, et al. (2014) Gut microbiota metabolism of dietary fiber influences allergic airway disease and hematopoiesis. Nat Med 20: 159-166.

52. Konijeti GG, Sauk J, Shrime MG, Gupta M, Ananthakrishnan AN (2014) Cost-effectiveness of competing strategies for management of recurrent Clostridium difficile infection: a decision analysis. Clin Infect Dis 58: 1507-1514. 